Published in final edited form as:

Adv Exp Med Biol. 2009 ; 656: 13-29.

\title{
Nuclear APC
}

\author{
Kristi L. Neufeld ${ }^{\star}$ \\ "Department of Molecular Biosciences, University of Kansas, Lawrence, KS, 66045 USA. \\ klneuf@ku.edu
}

\begin{abstract}
Mutational inactivation of the tumor suppressor gene APC (Adenomatous polyposis coli) is thought to be an initiating step in the progression of the vast majority of colorectal cancers. Attempts to understand APC function have revealed more than a dozen binding partners as well as several subcellular localizations including at cell-cell junctions, associated with microtubules at the leading edge of migrating cells, at the apical membrane, in the cytoplasm and in the nucleus. The present chapter focuses on APC localization and functions in the nucleus. APC contains two classical nuclear localization signals, with a third domain that can enhance nuclear import. Along with two sets of nuclear export signals, the nuclear localization signals enable the large APC protein to shuttle between the nucleus and cytoplasm. Nuclear APC can oppose $\beta$-cateninmediated transcription. This down-regulation of nuclear $\beta$-catenin activity by APC most likely involves nuclear sequestration of $\beta$-catenin from the transcription complex as well as interaction of APC with transcription corepressor CtBP. Additional nuclear binding partners for APC include transcription factor activator protein AP-2 $\alpha$, nuclear export factor Crm1, protein tyrosine phosphatase PTP-BL and perhaps DNA itself. Interaction of APC with polymerase $\beta$ and PCNA, suggests a role for APC in DNA repair. The observation that increases in the cytoplasmic distribution of APC correlate with colon cancer progression suggests that disruption of these nuclear functions of APC plays an important role in cancer progression. APC prevalence in the cytoplasm of quiescent cells points to a potential function for nuclear APC in control of cell proliferation. Clear definition of APC's nuclear function(s) will expand the possibilities for early colorectal cancer diagnostics and therapeutics targeted to APC.
\end{abstract}

\section{Introduction}

The tumor suppressor APC is $\sim 310 \mathrm{kDa}$ protein with minimal sequence homology to other characterized proteins. Mutation of the Apc gene is considered to be an early, if not the first step in the progression of more than $80 \%$ of all colorectal cancers, both inherited and sporadic. In an attempt to understand why APC function is so critical for suppression of colorectal cancers, several research groups used immunofluorescence microscopy to establish the subcellular localization pattern of APC in normal epithelial cells. The initial report characterizing APC in the cell's nucleus ${ }^{1}$ was received with some skepticism, due to previous reports of microtubule-associated APC at the leading edge of cells. ${ }^{2-4}$

Nevertheless, in the decade since the initial description of nuclear APC, research papers from more than 20 labs have confirmed the nuclear localization and have added to our understanding of the function of APC in the nucleus.

\section{Detection of Nuclear APC}

The first characterization of nuclear APC in human epithelial cells was published six years after identification of the APC gene. The nuclear APC localization was confirmed using four different APC antibodies and several cell lines for immunofluorescence microscopy and cell fractionation. ${ }^{1}$ Nuclear APC had previously been detected in the villus epithelial cells of 
normal mice as well as at the cell's periphery and throughout the cytoplasm. ${ }^{5}$ Subsequently, nuclear APC was also reported in normal human colon tissue as detected by confocal immunofluorescence microscopy ${ }^{6}$ and immunogold electron microscopy. ${ }^{7}$ In addition to the nuclear staining, prominent cell border staining was detected in nearly all of the images gathered from more than 50 patient samples. ${ }^{6}$ It is worth noting that in cultured cells, APC typically appears in the nucleus, in large puncta at the leading edge and in smaller puncta throughout the cytoplasm. In contrast, APC in normal human colon tissue appears in the nucleus and at the cell border, with little if any apparent cytoplasmic localization.

Initial examination of APC localization also revealed a nucleolar accumulation of APC in some cultured cells. ${ }^{1}$ Ectopically expressed full-length APC has also been described to concentrate in the nucleoli, especially with inhibition of nuclear export. ${ }^{8}$ Although observed using two independent methods, the nucleolar APC distribution appears rather transient and thus it remains unclear what role APC may have in the nucleoli. Regardless of the precise subnuclear localization, overwhelming data now support the idea that APC resides in the nucleus (Table 1a). ${ }^{1,5-8}$

\section{APC Domains Contributing to Nuclear Import and Export}

Nearly all $A P C$ mutations associated with colorectal cancer result in production of a truncated APC protein, typically including only the N-terminal 25-50\% of APC. In contrast to full-length APC, a truncated form of APC expressed in a colon cancer cell line did not display a nuclear distribution. ${ }^{1}$ Therefore, it was proposed that two potential nuclear localization signals (NLS) identified in the C-terminal half of APC and missing from the truncated form, were responsible for the different subcellular distributions of full-length versus truncated APC. At 2843 amino acids ( $\sim 310 \mathrm{kDa})$, APC is much too large to enter the nucleus by passive diffusion which has an estimated size limitation of $30-50 \mathrm{kDa} .{ }^{9}$ It is now known that APC contains two classic monopartite basic NLSs in the C-terminal half of the protein and at least one additional domain to facilitate its nuclear import (NLS-Arm, Figs. 1A, 1C, Table 1b). ${ }^{10,11}$ This additional domain of APC includes four of the seven Armadillo-repeat sequences comprising what is known as the ARM domain of APC (aa. 453-767, Fig. 1A). That the ARM domain could mediate nuclear import is not without precedence, as the $\beta$-catenin/Armadillo protein contains 12 Armadillo repeats and the three most C-terminal repeats, along with the C-terminal 116 aa tail appear to play an essential role in nuclear import of $\beta$-catenin. ${ }^{12}$ Nuclear import of $\beta$-catenin can occur in the absence of soluble factors or energy sources and has been proposed to be mediated through direct interaction with a nuclear pore component. ${ }^{13,14}$

Given that APC contains at least three nuclear localization domains, one might expect APC to be exclusively nuclear. Some potential explanations for cytoplasmic and cell junctional APC include regulation of the NLS activity, retention of APC at various locations by other protein partners and the presence of intrinsic nuclear export signals. In fact, all three of these factors likely influence the ultimate subcellular localization of APC.

As with nuclear import, proteins larger than 30-50 kDa cannot export through the nuclear pore by passive diffusion. Many proteins known to actively export from the nucleus contain leucine-rich amino acid sequences that mediate interaction with the nuclear export factor Crm1. APC contains five such classical nuclear export signals (NES), grouped at the Nterminus and in the central repeat region of the protein (Fig. 1A, C). The two NESs initially identified near the N-terminus of APC were shown to function using a wide variety of export assays (summarized in Table 1c). $8,15,16$

The identification of NESs in the central domain of APC was first made in Drosophila APC2/E-APC (dAPC2, Fig. 1B). At 1067 amino acids, dAPC2 is the smaller of the two 
Drosophila APC family members. dAPC2 shares $57 \%$ sequence identity with human APC in the ARM domain and contains similar features in the rest of the protein including 15-amino acid repeats, 20-amino acid repeats (20R) and SAMP repeats (Fig. 1B). ${ }^{17,18}$ Two NESs were identified in the 3rd and 4th 20R of dAPC $2 .{ }^{19}$ These are conserved in human APC which contains an additional putative NES in the 7th 20R (Figs. 1A, 1C). Evidence supporting the activity of these central NESs is also summarized in Table 1c. ${ }^{19,20}$

When all five NESs of human APC were analyzed side-by-side in a GFP-based nuclear export assay, NES1 appeared clearly dominant and it was proposed that NES1 is the primary NES in mediating nuclear export of APC. ${ }^{10}$ However, both of the N-terminal NESs appear active by a wide range of criteria (see Table 1c) and appear able to mediate nuclearcytoplasmic shuttling of truncated APC lacking the three 20R NESs. Alternatively, it has been argued that the middle 20R NESs predominantly control localization of full-length APC protein.20 The initial appeal of this hypothesis came from the observation that the 20R-NESs are eliminated from the truncated APC expressed in most colorectal cancers.19 This model predicts that truncated APC lacking the central NESs would be exportcompromised and nuclear. Indeed, APC truncated before NES-R3 was reported to be at least partially nuclear by immunofluorescence microscopic analysis of nine colorectal cancer cell lines. Consistent with this observation, cells expressing APC truncations that include NESR3 showed nuclear exclusion of APC.20 However, these results conflict directly with observations that endogenous truncated APC lacking NES-R3 found in SW480 colon cancer cells localizes predominantly in the cytoplasmic fraction, with a clear shift into the nuclear fraction following treatment with an inhibitor of Crm1-dependent nuclear export, leptomycin B (LMB).10,21 The conflicting results reported by these two groups could be attributed to differences in technique (immunofluorescence microscopy vs cell fractionation) and varying specificities of the APC antibodies used. The simplest explanation for all published results to date is that both NES regions influence the ultimate destination of fulllength APC protein, with one region dominating in particular circumstances and the other dominating under other conditions.

\section{APC Shuttles between the Nucleus and Cytoplasm}

The nearly concurrent reports from three independent labs that APC could shuttle between the nucleus and cytoplasm left little doubt that there was support for this conclusion. The detailed mechanism and the implications of APC nuclear shuttling have proven the biggest obstacles to reaching complete agreement by active researchers in this field.

If nuclear export of APC is Crm1-dependent, treating cells with LMB should result in an accumulation of APC in the nucleus. ${ }^{22}$ Indeed, full-length APC was more abundantly nuclear after LMB treatment in several different cell types, including human cervical carcinoma (HeLa) and human colon carcinoma (HCT116) cells, as determined by cell fractionation. ${ }^{15}, 21$ Furthermore, Drosophila APC2, while only approximately one-third the size of full-length human APC (Fig. 1B), showed apparent nuclear accumulation following LMB treatment of Drosophila embryos. ${ }^{19}$ Truncated forms of human APC also accumulated in the nucleus following LMB-treatment of colorectal cancer cell lines SW480 and HT29, suggesting that the nuclear-cytoplasmic shuttling ability of APC is not lost in some colorectal cancer cells. ${ }^{21}$ Localization of exogenously expressed full-length and truncated APC also appeared sensitive to LMB treatment, further supporting the conclusion that APC is capable of nucleo-cytoplasmic shuttling and that nuclear export is a CRM-1 dependent mechanism. ${ }^{8,10,23}$ 


\section{Factors that Effect the Subcellular Distribution of APC}

Sequence similarity between NLS2 ${ }_{\mathrm{APC}}$ and the well-characterized NLS in SV40 large T antigen led to the recognition that analogous regulation mechanisms may be at work, in particular, the ability to modify NLS activity through flanking phosphorylation sites. ${ }^{11,24}$ Indeed it appears that phosphorylation at a potential CK2 site upstream of NLS2 $\mathrm{APC}$ enhances nuclear import of APC; phosphorylation at a potential PKA site just downstream of NLS2 ${ }_{\mathrm{APC}}$ inhibits nuclear import (Fig. 2A).11,24 In addition, simultaneous exposure of cells to PKA agonists and CK2 inhibitors resulted in the anticipated accumulation of endogenous APC in the cytoplasm in both dog kidney epithelial (MDCK) and rat intestinal epithelial (IEC-6) cells. APC can be phosphorylated in vitro by both PKA ${ }^{25}$ and CK2,26 with association of endogenous CK2 and APC demonstrated by co-immunoprecipitation.26 Furthermore, in mouse keratinocytes where endogenous APC was predominantly nuclear as judged by cell fractionation, treatment with an inhibitor of p38MAPK, a kinase that activates CK2 and inhibits PKA, resulted in a redistribution of APC to the cytoplasm (Fig. 2B). ${ }^{27}$ The collective data indicates that nuclear APC levels are regulated, at least in part, by modification of $\mathrm{NLS}_{\mathrm{APC}}$ activity by phosphorylation.

Other clues regarding the function of APC in the nucleus have been found by examining various conditions that effect localization of APC. The subcellular distribution of both fulllength and truncated APC appears relatively constant as cells progress through the cell cycle.24,28 In contrast, canine kidney (MDCK) and rat intestinal (IEC6) epithelial cells grown to confluence and then maintained at high density for several days still displayed some nuclear APC, but exhibited more cytoplasmic APC than the subconfluent proliferating cells. ${ }^{24}$ A similar observation was made using thyroid carcinoma cells (C643), HEK293, C57MG and HCT116 cells, all of which express full-length APC.28,29 Together, this increase in cytoplasmic APC was visualized using five different APC antibodies for immunofluorescence microscopy.24,28,29 The increase in cytoplasmic APC levels only occurred after the cells were maintained in a confluent state for several days, suggesting that the increase was not a direct result of cell-cell contacts and might instead reflect a state of cellular quiescence. The observed increase in cytoplasmic APC was not dependent on sustained nuclear export, as LMB treatment did not induce a nuclear redistribution in the "super-confluent" cells. ${ }^{24} \mathrm{~A}$ fusion protein of a 32 amino acid stretch of APC containing NLS2 (aa. 2028-2058) with $\beta$-galactosidase displayed a cell density-influenced distribution similar to endogenous APC, suggesting that NLS2 can regulate this APC redistribution at high cell densities. ${ }^{24}$ Furthermore, alterations of the potential phosphorylation sites surrounding NLS2 attenuated this redistribution. Serum starvation of subconfluent MDCK cells also led to an increased number of cells with cytoplasmic APC coincident with decreased cellular proliferation. 28 Taken together, these observations imply that quiescent cells, although they maintain some nuclear APC, have less nuclear import of APC, leaving newly synthesized APC to accumulate in the cytoplasm. Thus, observed increases in cytoplasmic APC as cells stop proliferating and become quiescent likely result from reduced nuclear import, rather than increased export of nuclear APC to the cytoplasm.

Increased levels of cytoplasmic APC in quiescent confluent MDCK cells were reported by a fourth independent lab using a different APC antibody (M-APC). However, the authors argued that because cell fractionation experiments did not confirm this redistribution, the MAPC antibody might recognize a nonspecific nuclear epitope. ${ }^{21}$ Another possible interpretation, is that nuclear APC was associated with the nuclear matrix preventing its solubilization so that it was not collected in this particular cell fractionation assay.

Consistent with these observations in wild type cultured cells, variation in APC localization in proliferating versus quiescent cells has also been observed in human colon tissue. ${ }^{20} \mathrm{In}$ 
normal epithelia adjacent to the twenty one colon carcinomas analyzed, APC was predominantly cytoplasmic above the crypt (fully differentiated and quiescent cells), but both cytoplasmic and nuclear in the crypt where proliferating cells are located. Truncated APC also displays an increase in cytoplasmic distribution in SW480 colon cancer cells or KAT4 thyroid carcinoma cells grown beyond confluency. ${ }^{28,29}$ It was further noted that in large clusters of SW480 cells, peripheral cells displayed more truncated APC in the nucleus, while central cells showed more truncated APC at cell junctions.28 Both full-length and truncated APC were predominantly nuclear in proliferating cells, implicating nuclear APC in proliferation. Together, the data support a model in which the subcellular localization of APC reflects the proliferative status of the cell, with more cytoplasmic APC found in quiescent cells. Phosphorylation of sites near NLS2 ${ }_{\mathrm{APC}}$ might participate in control of this redistribution for full-length APC, whereas the truncated forms of APC must rely on other domains to facilitate this change in distribution.

Expanding on the evidence linking APC phosphorylation with cell cycle, full-length APC was found associated with and phosphorylated by casein kinase 2 (CK2) when normal fibroblast cells (TIG-1) were in $\mathrm{G}_{2} / \mathrm{M}$ with less association in $\mathrm{G}_{1} / \mathrm{S}$ and no association in $\mathrm{G}_{0} \cdot{ }^{26}$ This observation is compatible with the model where phosphorylation of APC by CK2 leads to activation of NLS2 $\mathrm{APC}$ and thus efficient nuclear import of APC. This nuclear import would in turn lead to an overall reduction in the cytoplasmic APC, consistent with a proliferative state (Fig. 2B). The model is further supported by the observation that inhibition of CK2 resulted in relocalization of endogenous APC from the nucleus to the cytoplasm in proliferating cells from two separate epithelial lines. ${ }^{24}$

The binding of APC to CK2 may also provide negative feedback to this system. Two different purified APC fragments (aa. 2086-2394 and 2518-2843) inhibited CK2 activity in vitro. ${ }^{26}$ Furthermore, constitutive expression of APC 2086-2394 strongly suppressed proliferation rates in two different cell lines and also impaired colony forming ability in soft agar.26 If the association of APC with CK2 inhibits the activity of CK2, the resulting reduction in nuclear import of APC might lead to increased cytoplasmic APC, decreased cell proliferation and ultimately cellular quiescence. Because truncated APC typically expressed in colon cancer cells lacks the ability to inhibit CK2 activity,26 these cancer cells would be expected to have impaired abilities to cease proliferation and enter quiescence.

The specific consequences of APC accumulation in the cytoplasm of quiescent cells remain to be defined, but the most obvious impact could be upon $\beta$-catenin signaling and cell cycle regulation. Increased levels of cytoplasmic APC could increase destruction complex activity and reduce $\beta$-catenin levels by targeting $\beta$-catenin for ubiquitin-mediated proteolysis. Nuclear APC might serve a critical role in proliferative cells, perhaps in DNA repair or transcription regulation as discussed below. In quiescent cells, nuclear APC might function in a different capacity, possibly by sequestering nuclear $\beta$-catenin from various transcription complexes and thereby inhibiting a Wnt signal, also discussed below.

\section{APC and Nuclear Export of $\beta$-Catenin}

To date, the best documented function of APC is to oppose a Wnt signal by acting in a complex with Axin, GSK3- $\beta$ and PP2A to target the protein $\beta$-catenin for proteasomemediated degradation in the cytoplasm in the absence of Wnt signaling. ${ }^{30}$ Because this important function of APC is covered in great detail elsewhere in this book (refer to Kennell and Cadigan, this volume) it will be only briefly summarized here. APC contains two types of $\beta$-catenin binding domains, the 15 aa and 20 aa repeats (Fig. 1A). The 15 aa repeats (aa. 1020-1169) are sufficient for $\beta$-catenin binding, whereas the 20 aa repeats (aa. 1262-2033) bind $\beta$-catenin and are required for its destruction. Given the well-characterized role for 
APC in $\beta$-catenin regulation and the knowledge that $\beta$-catenin serves as a transcription cofactor in the nucleus, several research groups have examined whether nuclear APC impacts $\beta$-catenin localization and activity. This regulation of nuclear $\beta$-catenin activity by APC could take the form of one or a combination of the following: enhanced nuclear export of $\beta$-catenin, nuclear sequestration of $\beta$-catenin, or repression of $\beta$-catenin's transcriptional activity.

Initial results implicated nuclear APC in mediating the nuclear export of $\beta$-catenin.8,19,23 In both $3 \mathrm{~T} 3$ and HCT116 cells, treatment with LMB resulted in a shift of endogenous $\beta$ catenin to the nucleus, suggesting that like APC, $\beta$-catenin shuttles between nucleus and cytoplasm. ${ }^{8}$ Exogenous expression of a truncated APC fragment (aa. 1-1309) which could still bind $\beta$-catenin, but couldn't target $\beta$-catenin for degradation resulted in a shift of endogenous $\beta$-catenin from the nucleus to the cytoplasm of SW480 cells. ${ }^{8}$ Blocking nuclear export of this exogenous APC, by either mutation of NES1 $1_{\mathrm{APC}}$ or LMB treatment abolished this cytoplasmic shift of $\beta$-catenin, thereby implicating APC in the nuclear export of $\beta$ catenin. Similarly, exogenous expression of full-length APC resulted in elimination of both cytoplasmic and nuclear $\beta$-catenin in SW480 cells, consistent with the well-characterized role for APC in $\beta$-catenin degradation. ${ }^{23}$ This reduction of $\beta$-catenin levels was abolished by mutation of both NES1 $1_{\mathrm{APC}}$ and NES2 $\mathrm{APC}$ or by LMB treatment. ${ }^{23}$ When nuclear export of APC was inhibited, both APC and the remaining $\beta$-catenin were predominantly nuclear. Nuclear export of APC containing nonfunctional NES1 APC and NES2 APC was restored by addition of a well-characterized NES sequence. This addition also restored APC-mediated degradation of endogenous $\beta$-catenin, demonstrating that the loss of APC's export function specifically affected $\beta$-catenin export and that the mutation of the N-terminal NESs in APC did not interfere with $\beta$-catenin binding or subsequent steps in $\beta$-catenin degradation. Together, these results suggest expression of APC with functional N-terminal NESs enhances nuclear export of $\beta$-catenin.

The 20R NESs of APC have also been implicated in nuclear export of $\beta$-catenin. ${ }^{19}$ When expressed in SW480 colon cancer cells, fragments of APC containing 20 aa repeats, SAMP repeats and the 20R NESs (aa. 1342-2075 or aa. 1379-2080) were sufficient to target endogenous $\beta$-catenin for destruction 31,19 and localized predominantly to the cytoplasm.19 LMB treatment or mutation of all three 20R-linked NESs increased the nuclear localization of the APC fragment and resulted in higher $\beta$-catenin levels.19,20 Nuclear $\beta$-catenin can serve as a transcription cofactor when bound to transcription factor LEF/TCF family members. Whereas expression of APC (aa 1379-2080) in SW480 cells resulted in reduced $\beta$ catenin transcriptional activity as measured using a standard reporter assay, mutation of one, two, or all three 20R-linked NESs resulted in partial restoration of the $\beta$-catenin transcription activity. ${ }^{19}$ Together, these results are consistent with a role for APC in mediating the nuclear export of $\beta$-catenin. However, it is likely that nuclear export of $\beta$ catenin can occur by multiple pathways since there is a solid body of evidence for nuclear export of $\beta$-catenin independent of APC and Crm1.14,32-34

\section{APC and Nuclear Sequestration of $\beta$-Catenin}

Many of the observations implicating APC in nuclear export of $\beta$-catenin are also consistent with nuclear APC dampening $\beta$-catenin activity by sequestration. ${ }^{23}$ Blocking nuclear export of exogenous full-length APC by either mutations in NES1 $1_{\mathrm{APC}}$ and $\mathrm{NES} 2_{\mathrm{APC}}$ or LMB treatment resulted in nuclear accumulation of both APC and endogenous $\beta$-catenin in SW480 colon cancer cells. ${ }^{23}$ Other components of the $\beta$-catenin destruction complex have been reported to be in the nucleus, with Axin also capable of nuclear export. ${ }^{35,36}$ However, since increasing levels of nuclear APC did not result in an apparent reduction in nuclear $\beta$ catenin, nuclear APC does not appear to target nuclear $\beta$-catenin for destruction within the 
nuclear compartment. ${ }^{23}$ It is of course possible that other components of the destruction complex are limiting in the nucleus so that excess APC cannot reduce $\beta$-catenin in this compartment. The unexpected finding that this abundant nuclear $\beta$-catenin was not active in standard $\beta$-catenin/LEF-1 activity assays led to the proposal that binding of nuclear $\beta$ catenin to APC sequesters $\beta$-catenin from LEF- 1 and renders $\beta$-catenin inactive as a transcription cofactor. ${ }^{23}$ Because APC and LEF-1 bind to the same domain of $\beta$-catenin, ${ }^{37}$ it was predicted that the binding of $\beta$-catenin to either protein is mutually exclusive.

Indeed, binding affinities of full-length $\beta$-catenin to an APC fragment containing 20R3 (aa. 1484-1528) that was homogenously phosphorylated by CK1 and GSK-3 $\beta$ in vitro or to fulllength LEF-1 as determined by isothermal titration calorimetry are $10 \mathrm{nM}$ and $20 \mathrm{nM}$, respectively. ${ }^{38}$ These binding affinities indicate that APC might effectively compete with LEF-1 for $\beta$-catenin binding, at least when APC is phosphorylated. Nonphosphorylated APC-R3 showed a reduced affinity to $\beta$-catenin by three orders of magnitude compared with phosphorylated APC-R3,38 thus providing a second example where APC phosphorylation influences the function of APC in the nucleus (Fig. 2C). A larger APC fragment containing 20R2 and 20R3 (aa. 1362-1540) also showed a 300-500 fold increase in binding affinity to $\beta$-catenin when APC was phosphorylated. ${ }^{39}$ Furthermore, this same phosphorylated APC fragment displaced TCF-3 bound to $\beta$-catenin in an in vitro binding assay, competed with a LEF-1/DNA complex for $\beta$-catenin binding in a mobility shift assay and inhibited $\beta$-catenin/ LEF-1 mediated transcription in vitro.40 While collectively these measurements reveal that phosphorylated APC competes effectively with LEF/TCF for $\beta$-catenin binding, recent data suggests phosphorylation of LEF- 1 by CK2 also enhances LEF- 1 binding to $\beta$-catenin.41

Together, these data support a model for regulation of Wnt signaling in the nucleus by means of a competition between APC and LEF- 1 for $\beta$-catenin binding that is dependent on the phosphorylation state of APC and LEF-1 (Fig. 2C). $\beta$-catenin interaction with phosphorylated APC is favored over a $\beta$-catenin/LEF-1 interaction. CK2 phosphorylation of LEF-1 increases the affinity of LEF-1 for $\beta$-catenin. APC binds to CK2 and renders it inactive, leaving LEF-1 in an unphosphorylated form and thereby less able to bind $\beta$-catenin. If nuclear APC functions by binding and sequestering $\beta$-catenin from the transcription complex, APC could very quickly dampen a Wnt signal once the Wnt ligand was no longer bound to its receptor. Nuclear export and subsequent cytoplasmic degradation of $\beta$-catenin would not be required for an immediate cellular response to removal of Wnt ligand. Consistent with this model, truncated APC found in most colon cancers binds to CK2 but can not inactivate it, thus leaving CK2 able to phosphorylate LEF-1. Truncated APC would not readily compete with the CK2-phosphorylated LEF-1 for $\beta$-catenin binding, leading to constitutive Wnt signaling in the nucleus of the colon cancer cells.

Consistent with a sequestration function of nuclear APC, endogenous APC and $\beta$-catenin coimmunoprecipitate from nuclear lysates of HCT116 colon cancer cells, using antibodies to either protein.23 Full-length APC expressed in SW480 cells in the presence of the proteasome-inhibiting drug MG132 colocalized with endogenous $\beta$-catenin in the nucleus, the cytoplasm and along the cytoskeleton, consistent with the notion that APC and $\beta$-catenin can interact in the nucleus. 8 Moreover, whereas ectopic expression of LEF-1 in mouse NIH 3 T3 fibroblasts led to $\beta$-catenin accumulation, co-expression of truncated APC (1-1309) which can bind $\beta$-catenin but not target it for destruction, reduced this LEF-1-induced increase in $\beta$-catenin levels. ${ }^{34}$ Additionally, in SW480 cells, ectopic expression of truncated APC (1-1309) also led to a reduction in $\beta$-catenin activity, even when this APC had a nonfunctional NES1. Similarly, expression of a GFP-tagged APC (aa. 1379-2080) with inactivating mutations in all three 20R-linked NESs still reduced endogenous $\beta$-catenin activity in SW480 colon cancer cells by half, suggesting that nuclear APC can influence $\beta$ catenin activity in the absence of nuclear export.20 
Initially reported as evidence against the sequestration model, SW480 cells showed a redistribution of otherwise predominantly nuclear $\beta$-catenin to the cytoplasm when APC (aa. 1-1309) was expressed ectopically.34 This cytoplasmic redistribution was blocked by further co-expression of LEF-1, suggesting APC could not compete effectively with LEF-1 for $\beta$-catenin binding. In light of the recent demonstration that full-length but not truncated APC can inhibit CK2, thereby reducing LEF-1's ability to bind $\beta$-catenin, the data gathered in the SW480 cells is consistent with the sequestration model. Therefore, APC binding to nuclear $\beta$-catenin and sequestering it from the LEF-1/TCF transcription complex remains a viable mechanism by which APC counteracts $\beta$-catenin. Moreover, recent evidence implicates nuclear retention rather than nuclear export as the dominant mechanism by which nuclear APC regulates nuclear $\beta$-catenin.42 Fluorescence recovery after photobleach (FRAP) of YFP-tagged $\beta$-catenin expressed in HEK293 cells, revealed highly efficient shuttling of $\beta$-catenin between nucleus and cytoplasm.42 Co-expression of APC with YFPtagged $\beta$-catenin slowed down this shuttling rather than accelerated it, suggesting that regulation of nuclear $\beta$-catenin by APC involves nuclear retention rather than active nuclear export.

\section{the Role of Other Nuclear Proteins in APC Mediated $\beta$-Catenin Sequestration}

There is growing evidence that other nuclear proteins might bind APC and modulate the ability of APC to displace $\beta$-catenin from the LEF-1/TCF complex. Transcription factor activator protein AP- $2 \alpha$ coprecipitates with full-length and truncated APC in three different cell lines ${ }^{43}$ and two different APC fragments (aa. $~ 1-400$ and $\sim 400-1000$ ) could bind AP-2 $\alpha$ in a GST pull down assay. Overexpression of AP- $2 \alpha$ in HEK293, HCT116 or HT29 cells appeared to decrease the association between TCF- 4 and $\beta$-catenin and decreased $\beta$-catenin activity. The fact that overexpressed AP- $2 \alpha$ did not associate with TCF-4, nor did it change the overall levels of nuclear $\beta$-catenin, suggests a role for AP- $2 \alpha$ in APC-mediated sequestration of nuclear $\beta$-catenin. The exact mechanism behind this modulation has yet to be determined.

The transcriptional corepressor C-terminal binding protein (CtBP) may also affect APCmediated $\beta$-catenin sequestration. CtBP was identified by mass spectrometry as a protein in crude Drosophila embryonic extract associated with Drosophila APC2-GST.44 Direct binding between human APC (aa. 918-1698) and CtBP was also demonstrated by GST-pull down assays and further, endogenous APC and CtBP were coprecipitated from 293T, HCT116 and SW480 cell lysates.44 A more recent report contradicts some of the initial findings regarding CtBP and APC interactions and showed that although full-length APC from human epithelial kidney cells (HEK-293) coprecipitated with endogenous CtBP, truncated APC representing roughly the N-terminal half of APC did not coprecipitate with CtBP in either of two colon cancer cell lines (HT29 and SW480).40 This casts doubt as to the precise APC domain responsible for CtBP binding. To test for the relevance of the APC$\mathrm{CtBP}$ interaction to nuclear $\beta$-catenin function, the sequestration of nuclear $\beta$-catenin was forced by expressing APC linked to an additional NLS resulting in a decrease in $\beta$-catenin activity. This decrease in activity was partially attenuated by alanine substitutions in the putative CtBP binding domain of APC, consistent with CtBP-binding enhancing the ability of APC to sequester nuclear $\beta$-catenin. Given the known functions of $\mathrm{CtBP}$, one might predict that APC delivers CtBP to the $\beta$-catenin/TCF complex where CtBP represses TCF activity. However, CtBP did not appear to coprecipitate with TCF-4 in the mammalian cells examined.44

Examination of the regulation of c-myc, a target gene downstream of Wnt signaling, has helped to shed light on the mechanisms involved in APC-CtBP nuclear function. ${ }^{40}$ To 
examine the dynamic nature of proteins bound to the c-myc enhancer element, various transcription coregulators were immunoprecipitated from mouse myoblast cells $(\mathrm{C} 2 \mathrm{C} 12)$ treated with lithium chloride (an inhibitor of GSK3 $\beta$ in the destruction complex) to increase $\beta$-catenin levels. The chromatin immunoprecipitation (ChIP) assay demonstrated a transient association of both $\beta$-catenin and APC with c-myc enhancer DNA accompanying this increase in $\beta$-catenin levels. Surprisingly, this study also showed that truncated APC associated with the c-myc enhancer in HT29 cells. Induction of full-length APC in HT29 cells resulted in a transient association of full-length APC with the c-myc enhancer DNA. A similar temporal association with the c-myc enhancer was shown for two other transcriptional corepressors, CtBP and Yin yang 1 (YY1), as well as for $\beta$-transducing repeat-containing protein $(\beta \operatorname{TrCP})$ which has been shown to target $\beta$-catenin for ubiquitination and subsequent degradation. The authors suggested that binding of the APC/ $\beta \mathrm{TrCP} / \mathrm{CtBP} / \mathrm{YY} 1$ complex to the c-myc enhancer leads to rapid repression of c-myc transcription. Of note in this set of experiments, APC was associated with DNA at times when $\beta$-catenin was not, revealing that APC might regulate transcription by multiple mechanisms, not merely by displacing $\beta$-catenin from the LEF-1/TCF complex. Overall, these experiments indicated a similar temporal association of APC and transcription corepressors with the chromatin of a Wnt-responsive gene. Thus, it is likely that APC opposes a Wnt signal not only by targeting cytoplasmic $\beta$-catenin for destruction, but also by binding and sequestering $\beta$-catenin and by associating with corepressors at the transcription complex (Fig. 3). Future experiments will surely clarify the broad range of APC activities as they relate to transcription control.

\section{Interaction of APC with DNA and Other Nuclear Proteins}

There is preliminary evidence for a direct interaction between APC and DNA. A $180 \mathrm{kDa}$ protein recognized by antibodies raised against both the $\mathrm{N}$ - and C-terminal region of APC was identified in the soluble nuclear fraction of both HEK-293 and HCT116 cells. ${ }^{45}$ Although it is puzzling that an APC truncation would retain both $\mathrm{N}$ - and $\mathrm{C}$-terminal regions, three recombinant protein fragments, all from the C-terminal half of APC, bound plasmid DNA in mobility shift assays. Repetitive preparative mobility shift assays using two of these APC fragments revealed no consensus DNA binding sequence, but did suggest a higher affinity for $\mathrm{A} / \mathrm{T}$ nucleotides vs $\mathrm{G} / \mathrm{C}$.

In addition to DNA, other nuclear proteins can interact with APC. Full length APC from HCT116 colon cancer cells can bind polymerase $\beta$ and PCNA, part of the base excision repair (BER) pathway, raising the intriguing possibility that APC participates in DNA repair. ${ }^{46}$ The APC fragment (aa. 1250-1269) capable of polymerase $\beta$ and PCNA binding contains a PCNA-interacting protein-like box (PIP-like box). The PIP-like domain of APC appeared to block repair of a multi-nucleotide repair patch, "long patch BER", but not a damaged single nucleotide base, "short patch BER", in vitro.

Furthermore, APC also interacts with protein tyrosine phosphatase (PTP-BL) in the nucleus. The C-terminal 19 amino acids of APC appear to mediate binding to PTP-BL, with a dissociation constant of $8 \mathrm{nM}$ as measured using surface plasmon resonance analysis. ${ }^{47}$ APC and PTP-BL colocalized both at the tips of cellular extensions and in the nucleus of MDCK cells, once again implicating phosphorylation as a means to regulate nuclear function(s) of APC. The exact roles of these additional nuclear interactions of APC and whether they impinge upon Wnt signal transduction will require further investigation.

\section{The Requirement for Nuclear APC in Tumor Suppression}

There is limited evidence supporting a role for nuclear APC in tumor suppression in the colon. The vast majority of germline and sporadic APC mutations in colorectal cancers 
result in protein truncation, leaving only the N-terminal half of the APC protein expressed. Although lacking the central NLSs and NESs, this truncated APC protein typically retains the ability to shuttle and thus could negatively impact nuclear processes that depend on the C-terminus of APC. In human colon tissue, truncated APC protein as detected with Nterminal APC antibodies remained strongly nuclear in polyps and carcinomas. ${ }^{6}$ However, the frequency of cytoplasmic APC progressively increased, with $60 \%$ of the carcinoma tissues showing cytoplasmic APC compared to only $4 \%$ of the normal tissues sampled. ${ }^{6}$ Since inactivating APC mutations are found in over $80 \%$ of all colon carcinomas, most carcinoma tissue should display no full-length APC. Indeed, of the 148 images of human carcinoma tissue analyzed, only $11 \%$ showed nuclear staining using C-terminal APC antibodies. ${ }^{6}$ Although they represent a minority, there are several germline missense mutations found in APC. Of note, a few of these mutations result in amino acid substitutions in or near the NLS and NES domains. While the missense mutations indicate a potential requirement for these domains in APC-mediated tumor suppression, to date, none of these amino acid substitutions have been examined for their impact on NLS and NES activity. Overall, the changes in APC localization as human colon tissue progresses from normal to polyp to carcinoma and the germline mutations found in and near APC's nuclear import and export signals are consistent with a role for nuclear APC in tumor suppression.

In addition to colon tissue, nuclear APC has been described in alveolar soft-part sarcoma and in over $62 \%$ of 92 small cell lung cancers (SCLC) sampled. ${ }^{48,49}$ Besides the human and mouse studies, nuclear APC has been described in cells from worm,50 frog,51 fruit fly, 19 monkey52 and dog.24 At present, mouse models have not been particularly informative in determining the nuclear function of APC. Mice expressing only a truncated form of APC (aa. 1-1638) had reduced viability, but those surviving to adulthood had no increase in intestinal polyp number compared to wild-type mice. ${ }^{53}$ This surprising finding is consistent with the observation that APC-interacting proteins $\beta$-tubulin, EB-1 and discs large (DLG) retained normal subcellular localization patterns in these mice and in fact, the truncated APC remained nuclear. The ARM domain still retained in this truncated APC likely contributed to nuclear import. The definitive genetic test for nuclear function of APC in a model organism has yet to be completed, but entails abrogation of the nuclear import ability of APC in a mouse model.

\section{Future Applications, New Research, and Anticipated Developments}

Although there are many areas of consensus in the field of nuclear APC localization and function, there have also been some conflicting results with no apparent resolution. Experiments performed with protein fragments of APC over-expressed in a few colon cancer cell lines have led to speculation about the relative activity of various NESs and NLSs. One complication in interpreting such results is that functions ascribed to small fragments of APC might not accurately reflect functions when these sequences are in the context of full-length native APC. In addition, these exogenous APC fragments are often over-expressed compared to endogenous APC levels and thus might saturate binding to protein partners in limited supply. Finally, there appears to be no readily available normal human colon epithelial cell line, nor a cell line completely lacking APC. In the absence of such model cell lines, most experiments are performed using colon cancer cell lines that express endogenous APC, either full-length or truncated. Because truncated APC still retains an $\mathrm{N}$-terminal oligomerization domain, ${ }^{54}$ there is the potential for heterodimerization between full-length and truncated APC molecules, possibly confounding results.

Unfortunately, complexity is also encountered when endogenous APC is examined. The specificity of several APC antibodies has been questioned, with no clear consensus about the "best" antibody to use for APC detection. ${ }^{21,55-57}$ With the potential specificity issues 
surrounding APC detection by immunofluorescence microscopy, one might think that cellular fractionation followed by immunoblot would serve as a better "gold standard" for APC distribution. Regrettably, use of different fractionation protocols leads to considerable variation in the distribution of soluble nuclear proteins and cytoskeletal/membrane associated proteins and potential elimination of nuclear matrix-associated insoluble proteins, making cell fractionation followed by immunoblot analysis potentially problematic as well.

The best solution appears to be analysis of APC using several complimentary techniques and a variety of APC antibodies with the least amount of demonstrated nonspecific cross reactivity, with quantitation whenever possible.

This is an exciting time for researchers interested in nuclear functions of APC. APC has been identified in the nucleus of many different cultured cell lines as well as in normal human colon tissue and tissue from other organisms. Intrinsic localization signals have been characterized that facilitate shuttling of APC between the nucleus and cytoplasm.

Phosphorylation of APC provides one underlying mechanism by which APC localization and activity are regulated. APC distribution within a cell appears to correlate with the proliferative or quiescent status of the cell. Several intriguing nuclear binding partners have already been identified, with consequences of APC binding to these nuclear proteins in preliminary stages. For example, hints of a direct link between APC and the transcription complex or the DNA repair machinery are beginning to emerge. APC likely serves to regulate Wnt signaling, in part, by competing with nuclear LEF-1 for $\beta$-catenin binding. It is anticipated that future work will clarify the role of APC in association with various proteins at the c-myc enhancer and potentially as APC interacts directly with DNA. Future biochemical analysis is expected to reveal additional nuclear proteins that interact with APC, as well as the cellular consequences of APC association with these proteins. The definitive test to assess the requirement of nuclear APC in suppression of colorectal tumors will be to eliminate nuclear APC from a model organism. One such mouse model currently in production should prove useful to address some of the remaining questions. Ultimately, a more thorough understanding of the many functions of the tumor suppressor APC will ultimately lead to better diagnostics and more targeted therapeutics for colorectal cancer.

\section{Acknowledgments}

The author would like to thank Drs. T. Chris Gamblin, Audrey Lamb and Erik Lundquist for critical review of the manuscript and Jamie Cunningham for contributions to Figure 1. The author also thanks Drs. Brooke McCartney and Inke Nathke for soliciting this chapter and for their helpful editorial suggestions. The author wishes to acknowledge the support of the National Cancer Institute/National Institute of Health (RO1CA109220-01).

Apologies are extended to colleagues whose research was omitted from the review due to size limitations.

\section{References}

1. Neufeld KL, White RL. Nuclear and cytoplasmic localizations of the adenomatous polyposis coli protein. Proc Natl Acad Sci USA. 1997; 94:3034-3039. [PubMed: 9096341]

2. Munemitsu S, Souza B, Muller O, Albert I, Rubinfeld B, Polakis P. The APC gene product associates with microtubules in vivo and promotes their assembly in vitro. Cancer Research. 1994; 54(14):3676-3681. [PubMed: 8033083]

3. Smith K, Levy D, Maupin P, Pollard T, Vogelstein B, Kinzler K. Wild-type but not mutant APC associates with the microtubule cytoskeleton. Cancer Research. 1994; 54(14):3672-3675. [PubMed: 8033082]

4. Nathke IS, Adams CL, Polakis P, Sellin JH, Nelson J. The adenomatous polyposis coli tumor suppressor protein localizes to plasma membrane sites involved in active cell migration. J. Cell Biol. 1996; 134:165-179. [PubMed: 8698812] 
5. Wong MH, Hermiston ML, Syder AJ, Gordon JI. Forced expression of the tumor suppressor adenomatosis polyposis coli protein induces disordered cell migration in the intestinal epithelium. Proc Natl Acad Sci U S A. 1996; 93(18):9588-9593. [PubMed: 8790374]

6. Anderson CB, Neufeld KL, White RL. Subcellular distribution of Wnt pathway proteins in normal and neoplastic colon. Proc Natl Acad Sci U S A. Jun 25; 2002 99(13):8683-8688. [PubMed: 12072559]

7. Sena P, Saviano M, Monni S, Losi L, Roncucci L, Marzona L, Pol AD. Subcellular localization of beta-catenin and APC proteins in colorectal preneoplastic and neoplastic lesions. Cancer Lett. Sep 28; 2006 241(2):203-212. [PubMed: 16298038]

8. Henderson B. Nuclear-cytoplasmic shuttling of APC regulates $\beta$-catenin subcellular localization and turnover. Nat Cell Biol. 2000; 2:653-660. [PubMed: 10980707]

9. Paine PL, Moore LC, Horowitz SB. Nuclear envelope permeability. Nature. Mar 13; 1975 254(5496):109-114. [PubMed: 1117994]

10. Galea MA, Eleftheriou A, Henderson BR. ARM domain-dependent nuclear import of adenomatous polyposis coli protein is stimulated by the B56 alpha subunit of protein phosphatase 2A. J Biol Chem. 2001; 276(49):45833-45839. [PubMed: 11585828]

11. Zhang F, White R, Neufeld K. Phosphorylation near nuclear localization signal regulates nuclear import of APC protein. Proc. Natl. Acad. USA. 2000; 97:12577-12582.

12. Koike M, Kose S, Furuta M, Taniguchi N, Yokoya F, Yoneda Y, Imamoto N. beta-Catenin shows an overlapping sequence requirement but distinct molecular interactions for its bidirectional passage through nuclear pores. J Biol Chem. Aug 6; 2004 279(32):34038-34047. [PubMed: 15173161]

13. Yokoya F, Imamoto N, Tachibana T, Yoneda Y. beta-catenin can be transported into the nucleus in a Ran-unassisted manner. Mol Biol Cell. 1999; 10(4):1119-1131. [PubMed: 10198061]

14. Wiechens N, Fagotto F. CRM1- and Ran-independent nuclear export of beta-catenin. Curr Biol. 2001; 11(1):18-27. [PubMed: 11166175]

15. Neufeld KL, Nix DA, Bogerd H, Kang Y, Beckerle MC, Cullen BR, White RL. Adenomatous Polyposis Coli Protein Contains Two Nuclear Export Signals and Shuttles between the Nucleus and Cytoplasm. Proc Natl Acad Sci USA. 2000; 97:12085-12090. [PubMed: 11035805]

16. Tickenbrock L, Cramer J, Vetter IR, Muller O. The coiled coil region (amino acids 129-250) of the tumor suppressor protein adenomatous polyposis coli (APC). Its structure and its interaction with chromosome maintenance region 1 (Crm-1). J Biol Chem. Aug 30; 2002 277(35):32332-32338. [PubMed: 12070164]

17. McCartney BM, Dierick HA, Kirkpatrick C, Moline MM, Baas A, Peifer M, Bejsovec A. Drosophila APC2 is a cytoskeletally-associated protein that regulates wingless signaling in the embryonic epidermis. J Cell Biol. 1999; 146(6):1303-1318. [PubMed: 10491393]

18. Yu X, Waltzer L, Bienz M. A new Drosophila APC homologue associated with adhesive zones of epithelial cells. Nat Cell Biol. 1999; 1(3):144-151. [PubMed: 10559900]

19. Rosin-Arbfeld R, Townsley F, Bienz M. The APC tumour suppressor has a nuclear export function. Nature. 2000; 406:1009-1012. [PubMed: 10984057]

20. Rosin-Arbesfeld R, Cliffe A, Brabletz T, Bienz M. Nuclear export of the APC tumour suppressor controls beta-catenin function in transcription. Embo J. Mar 3; 2003 22(5):1101-1113. [PubMed: 12606575]

21. Brocardo M, Nathke IS, Henderson BR. Redefining the subcellular location and transport of APC: new insights using a panel of antibodies. EMBO Rep. Feb; 2005 6(2):184-190. [PubMed: 15678162]

22. Fukuda M, Asano S, Nakamura T, Adachi M, Yoshida M, Yanagida M, Nishida E. CRM1 is responsible for intracellular transport mediated by the nuclear export signal. Nature. 1997; 390(6657):308-311. [PubMed: 9384386]

23. Neufeld KL, Zhang F, Cullen BR, White RL. APC-mediated down-regulation of $\beta$-Catenin activity involves nuclear sequestration and nuclear export. EMBO Reports. 2000; 6:519-523. [PubMed: 11263497] 
24. Zhang F, White RL, Neufeld KL. Cell density and phosphorylation control the subcellular localization of adenomatous polyposis coli protein. Mol Cell Biol. 2001; 21(23):8143-8156. [PubMed: 11689703]

25. Rubinfeld B, Albert I, Porfiri E, Fiol C, Munemitsu S, Polakis P. Binding of GSK3 $\beta$ to the APC- $\beta$ catenin complex and regulation of complex assembly. Science. 1996; 272:1023-1026. [PubMed: 8638126]

26. Homma MK, Li D, Krebs EG, Yuasa Y, Homma Y. Association and regulation of casein kinase 2 activity by adenomatous polyposis coli protein. Proc Natl Acad Sci U S A. Apr 30; 2002 99(9): 5959-5964. [PubMed: 11972058]

27. Hildesheim J, Salvador JM, Hollander MC, Fornace AJ Jr. Casein kinase 2- and protein kinase Aregulated adenomatous polyposis coli and beta-catenin cellular localization is dependent on p38 MAPK. J Biol Chem. Apr 29; 2005 280(17):17221-17226. [PubMed: 15649893]

28. Fagman H, Larsson F, Arvidsson Y, Meuller J, Nordling M, Martinsson T, Helmbrecht K, Brabant G, Nilsson M. Nuclear accumulation of full-length and truncated adenomatous polyposis coli protein in tumor cells depends on proliferation. Oncogene. Sep 4; 2003 22(38):6013-6022. [PubMed: 12955080]

29. Davies ML, Roberts GT, Spiller DG, Wakeman JA. Density-dependent location and interactions of truncated APC and beta-catenin. Oncogene. Feb 19; 2004 23(7):1412-1419. [PubMed: 14647421]

30. Giles RH, van Es JH, Clevers H. Caught up in a Wnt storm: Wnt signaling in cancer. Biochim Biophys Acta. Jun 5; 2003 1653(1):1-24. [PubMed: 12781368]

31. Munemitsu S, Albert I, Souza B, Rubinfeld B, Polakis P. Regulation of intracellular beta-catenin levels by the adenomatous polyposis coli (APC) tumor-suppressor protein. Proc Natl Acad Sci USA. 1995; 92(7):3046-3050. [PubMed: 7708772]

32. Eleftheriou A, Yoshida M, Henderson BR. Nuclear export of human beta-catenin can occur independent of CRM1 and the adenomatous polyposis coli tumor suppressor. J Biol Chem. Jul 13; 2001 276(28):25883-25888. [PubMed: 11337505]

33. Hendriksen J, Fagotto F, van der Velde H, van Schie M, Noordermeer J, Fornerod M. RanBP3 enhances nuclear export of active (beta)-catenin independently of CRM1. J Cell Biol. Dec 5; 2005 171(5):785-797. [PubMed: 16314428]

34. Henderson BR, Galea M, Schuechner S, Leung L. Lymphoid enhancer factor-1 blocks adenomatous polyposis coli-mediated nuclear export and degradation of beta-catenin. Regulation by histone deacetylase 1. J Biol Chem. Jul 5; 2002 277(27):24258-24264. [PubMed: 11986304]

35. Cong F, Varmus H. Nuclear-cytoplasmic shuttling of Axin regulates subcellular localization of beta-catenin. Proc Natl Acad Sci U S A. Mar 2; 2004 101(9):2882-2887. [PubMed: 14981260]

36. Wiechens N, Heinle K, Englmeier L, Schohl A, Fagotto F. Nucleo-cytoplasmic shuttling of Axin, a negative regulator of the Wnt-beta-catenin Pathway. J Biol Chem. Feb 13; 2004 279(7):52635267. [PubMed: 14630927]

37. Orsulic S, Huber O, Aberle H, Arnold S, Kemler R. E-cadherin binding prevents beta-catenin nuclear localization and beta-catenin/LEF-1-mediated transactivation. J Cell Sci. 1999; 112(Pt 8): 1237-1245. [PubMed: 10085258]

38. Choi HJ, Huber AH, Weis WI. Thermodynamics of beta-catenin-ligand interactions: the roles of the N- and C-terminal tails in modulating binding affinity. J Biol Chem. Jan 13; 2006 281(2): 1027-1038. [PubMed: 16293619]

39. Xing Y, Clements WK, Le Trong I, Hinds TR, Stenkamp R, Kimelman D, Xu W. Crystal structure of a beta-catenin/APC complex reveals a critical role for APC phosphorylation in APC function. Mol Cell. Aug 27; 2004 15(4):523-533. [PubMed: 15327769]

40. Sierra J, Yoshida T, Joazeiro CA, Jones KA. The APC tumor suppressor counteracts beta-catenin activation and H3K4 methylation at Wnt target genes. Genes Dev. Mar 1; 2006 20(5):586-600. [PubMed: 16510874]

41. Wang S, Jones KA. CK2 controls the recruitment of Wnt regulators to target genes in vivo. Curr Biol. Nov 21; 2006 16(22):2239-2244. [PubMed: 17113388]

42. Krieghoff E, Behrens J, Mayr B. Nucleo-cytoplasmic distribution of beta-catenin is regulated by retention. J Cell Sci. Apr 1; 2006 119(Pt 7):1453-1463. [PubMed: 16554443] 
43. Li Q, Dashwood RH. Activator protein 2alpha associates with adenomatous polyposis coli/betacatenin and Inhibits beta-catenin/T-cell factor transcriptional activity in colorectal cancer cells. J Biol Chem. Oct 29; 2004 279(44):45669-45675. [PubMed: 15331612]

44. Hamada F, Bienz M. The APC tumor suppressor binds to C-terminal binding protein to divert nuclear beta-catenin from TCF. Dev Cell. Nov; 2004 7(5):677-685. [PubMed: 15525529]

45. Deka J, Herter P, Sprenger-Haussels M, Koosch S, Franz D, Muller KM, Kuhnen C, Hoffmann I, Muller O. The APC protein binds to A/T rich DNA sequences. Oncogene. 1999; 18(41):56545661. [PubMed: 10523845]

46. Narayan S, Jaiswal AS, Balusu R. Tumor suppressor APC blocks DNA polymerase beta-dependent strand displacement synthesis during long patch but not short patch base excision repair and increases sensitivity to methylmethane sulfonate. J Biol Chem. Feb 25; 2005 280(8):6942-6949. [PubMed: 15548520]

47. Erdmann KS, Kuhlmann J, Lessmann V, Herrmann L, Eulenburg V, Muller O, Heumann R. The Adenomatous Polyposis Coli-protein (APC) interacts with the protein tyrosine phosphatase PTPBL via an alternatively spliced PDZ domain. Oncogene. 2000; 19(34):3894-3901. [PubMed: 10951583]

48. Kuhnen C, Herter P, Monse H, Kahmann S, Muehlberger T, Vogt PM, Steinau HU, Muller KM, Muller O. APC and beta-catenin in alveolar soft part sarcoma (ASPS)--immunohistochemical and molecular genetic analysis. Pathol Res Pract. 2000; 196(5):299-304. [PubMed: 10834386]

49. Kotsinas A, Evangelou K, Zacharatos P, Kittas C, Gorgoulis VG. Proliferation, but not apoptosis, is associated with distinct beta-catenin expression patterns in non-small-cell lung carcinomas: relationship with adenomatous polyposis coli and $\mathrm{G}(1)$-to S-phase cell-cycle regulators. Am J Pathol. Nov; 2002 161(5):1619-1634. [PubMed: 12414510]

50. Mizumoto K, Sawa H. Cortical beta-catenin and APC regulate asymmetric nuclear beta-catenin localization during asymmetric cell division in C. elegans. Dev Cell. Feb; 2007 12(2):287-299. [PubMed: 17276345]

51. Klymkowsky MW, Williams BO, Barish GD, Varmus HE, Vourgourakis YE. Membrane-anchored plakoglobins have multiple mechanisms of action in Wnt signaling. Mol Biol Cell. 1999; 10(10): 3151-3169. [PubMed: 10512857]

52. Langford KJ, Lee T, Askham JM, Morrison EE. Adenomatous polyposis coli localization is both cell type and cell context dependent. Cell Motil Cytoskeleton. Aug; 2006 63(8):483-492. [PubMed: 16767746]

53. Smits R, Kielman MF, Breukel C, Zurcher C, Neufeld K, Jagmohan-Changur S, Hofland N, van Dijk J, White R, Edelmann W, Kucherlapati R, Khan PM, Fodde R. Apc1638T: a mouse model delineating critical domains of the adenomatous polyposis coli protein involved in tumorigenesis and development. Genes Dev. 1999; 13(10):1309-1321. [PubMed: 10346819]

54. Joslyn G, Richardson D, White R, Alber T. Dimer formation by an N-terminal coiled coil in the APC protein. Proc Nat Acad Sci USA. 1993; 90:11109-11113. [PubMed: 8248216]

55. Roberts GT, Davies ML, Wakeman JA. Interaction between Ku80 protein and a widely used antibody to adenomatous polyposis coli. Br J Cancer. Jan 27; 2003 88(2):202-205. [PubMed: 12610503]

56. Rosin-Arbesfeld R, Ihrke G, Bienz M. Actin-dependent membrane association of the APC tumour suppressor in polarized mammalian epithelial cells. Embo J. Nov 1; 2001 20(21):5929-5939. [PubMed: 11689433]

57. Davies ML, Roberts GT, Stuart N, Wakeman JA. Analysis of a panel of antibodies to APC reveals consistent activity towards an unidentified protein. Br J Cancer. 2007; 97(3):384-390. [PubMed: 17595655] 


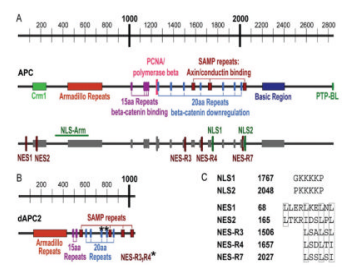

Figure 1.

Schematic representation of APC domains as they relate to nuclear functions. A) Human APC is 2843 amino acids, with binding sites for several proteins shown on the middle panel. The 15 aa repeats $(15 R)$ and 20 aa repeats (20R) are involved in $\beta$-catenin binding and degradation. The lower panel highlights regions of APC that facilitate nuclear-cytoplasmic shuttling. Two NESs are located near the N-terminus, with three more in the 20aa repeat domain in the center of APC. Two NLSs are located in the central domain near axin binding sites. An additional region of APC that is able to facilitate nuclear import of truncated APC is shown, NLS-Arm. B) Drosophila APC2 (dAPC2) is 1067 amino acids, with conserved domains labeled and NES-R3 and -R4 indicated (*). Figures are to scale, with amino acid increments indicated at the top. C) Nuclear localization signals and nuclear export sequences in human APC. Numbers for the initial amino acid in each sequence are shown. Boxes indicate important hydrophobic residues (leucine or isoleucine). 


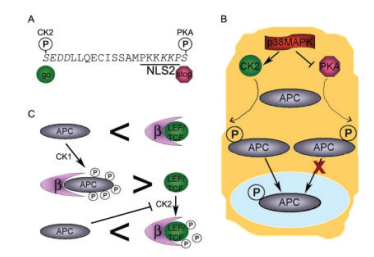

Figure 2.

Phosphorylation of APC regulates activity. A) The activity of NLS2APC contributes to nuclear import of APC. While phosphorylation of the serine upstream of NLS2 results in a more active NLS, phosphorylation of the serine just adjacent and downstream of NLS2 inhibits NLS activity. Potential CK2 and PKA recognition sequences are shown in italics. B) p38 MAPK activates CK2 and inhibits PKA. Inhibition of p38MAPK results in reduced levels of nuclear APC. C) LEF/TCF and APC compete for $\beta$-catenin binding. $\beta$-catenin binds LEF/TCF with a higher affinity than unphosphorylated APC. In contrast, $\beta$-catenin binds CK1-phosphorylated APC with a higher affinity than LEF/TCF. CK2-phosphorylated LEF-1 shows stronger binding to $\beta$-catenin, but APC can inhibit CK2 activity. 


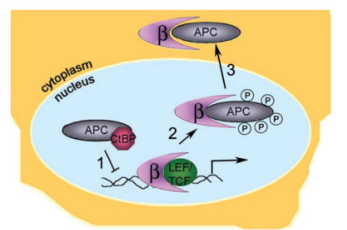

Figure 3.

Model for the roles of nuclear APC in regulation of Wnt target genes. APC modifies expression of Wnt target genes by three mechanisms. 1) APC associates with transcription corepressors, such as $\mathrm{CtBP}$, potentially guiding them to the transcription complex. 2) Phosphorylated APC competes with LEF/TCF for $\beta$-catenin binding, thus displacing $\beta$ catenin from the transcription complex. 3) APC may assist in the nuclear export of $\beta$-catenin for cytoplasmic degradation. 
Table 1a

Evidence for nuclear APC

\begin{tabular}{llll}
\hline Observation & Cell line(s) & Antibodies & Ref \\
\hline APC in nuclear puncta by immunofluorescence (IF) & 184A1, T47D & Ab-2,-4,-6, APC64 & 1 \\
$\begin{array}{l}\text { microscopy (also cytoplasmic and at leading edge) } \\
\text { Cell fractionation/immunoblot: }\end{array}$ & LS174T, HCT116 & Ab-1,-2 & 1 \\
$\begin{array}{l}\text { APC in nuclear and nuclear matrix fractions } \\
\text { truncated APC not in nuclear fractions }\end{array}$ & DLD-1 & Ab-1 & 1 \\
APC in nuclei of murine villus epithelial cells & mouse tissue & a-APC2 (middle) & 5 \\
$\begin{array}{l}\text { IF with confocal microscopy } \\
\text { APC in nuclei of human colonic epithelial cells }\end{array}$ & human tissue, & 10 different & 6 \\
IF with confocal microscopy & 50 patient samples & & \\
APC in nuclei of human colonic epithelial cells & human tissue & C-APC 28.9 & 7 \\
$\begin{array}{l}\text { Immunogold electron microscopy } \\
\text { Nuclear APC colocalizes with rRNA (nucleoli) }\end{array}$ & 184A1 & Ab-4 & 1 \\
APC in nucleoli when ectopically expressed, and & 3T3, HCT116 & Ab-7 & 8 \\
nuclear export inhibited by leptomycin B (LMB) & & & \\
\hline
\end{tabular}


Table 1b

Evidence for APC's nuclear import signals (NLS)

\begin{tabular}{|c|c|c|}
\hline & & Ref \\
\hline \multicolumn{2}{|c|}{ NIS1 (aa. 1767-1772) } & \multirow{4}{*}{11} \\
\hline 1 & Targets heterologous cytoplasmic protein $\beta$-Gal for nuclear import alanine substitutions for all 4 lysines abolished this ability & \\
\hline 2 & 2 NLS1 fused in tandem mediated nuclear import more efficiently than one & \\
\hline 3 & Mutation in context of full-length APC compromises nuclear import & \\
\hline \multicolumn{2}{|c|}{ NIS2 (aa. 2048-2053) } & \multirow{4}{*}{11} \\
\hline 1 & $\begin{array}{l}\text { Targets heterologous cytoplasmic protein } \beta \text {-Gal for nuclear import alanine substitutions for first } 2 \text { lysines abolished this } \\
\text { ability }\end{array}$ & \\
\hline 2 & Mutation in context of full-length APC compromises nuclear import & \\
\hline 3 & $\begin{array}{l}\text { Activity blocked by phosphorylation of potential PKA site and enhanced by phosphorylation of flanking CDK2 site in NLS- } \\
\beta \text {-Gal fusion and in full-length APC, exogenously expressed }\end{array}$ & \\
\hline \multicolumn{3}{|c|}{ NIS-Arm (aa. 334-625) } \\
\hline 1 & Endogenous truncated APC lacking NLS1 and NLS2 can localize to the nucleus & \multirow[t]{2}{*}{10} \\
\hline 2 & $\begin{array}{l}\text { Truncated APC can localize to the nucleus when expressed exogenously, nuclear localization was enhanced with co- } \\
\text { expression of B56 } \alpha \text { although not dependent on phosphatase activity }\end{array}$ & \\
\hline
\end{tabular}


Table 1c

Evidence for APC's nuclear export signals (NES)

Ref

NES1 (aa. 68-77) and NES2 (aa. 165-174)

1. Each directs nuclear export when fused to GFP-tagged HIV-Rev with inactivated endogenous NES

2. Each directs nuclear export when fused to purified GST and injected into nuclei of living cells

3. Each can functionally substitute for HIV-Rev NES in in vivo Rev activity assay

Together, mediated Crm1 interaction with a short $\mathrm{N}$-terminal fragment of APC (aa. 1-270) in a mammalian two-hybrid assay

5. The two NESs were able to facilitate nuclear shuttling of APC fragment (aa 1-270) in a 15 heterokaryon assay

6. Substitution of alanine for critical leucine residues in each NES inhibited the activities measured in all assays listed (1-5)

7. Mutation of either NES in the context of full-length exogenous APC resulted in shift of APC from predominantly cytoplasmic to more nuclear than cytoplasmic

Purified monomeric fragment of APC (aa. 129-250) containing only NES2 bound the nuclear exportin protein $\mathrm{Crm} 1$ in vitro

NES-R3 (aa. 1506-1511), NES-R4 (aa. 1657-1662) and NES-R7 (aa. 2027-2032)

1. Each directs nuclear export when fused to GFP-tagged N-terminal fragment of 19 Drosophila APC2, dAPC2 (aa. 114-410)

2. dAPC2 (aa. 460-1067) containing NES-R3 and -R4 and fused with GFP was cytoplasmic when expressed in COS cells, but more nuclear when cells were treated with LMB

3. APC (aa. 1379-2080) containing NES-R3, -R4 and -R7 behaved similarly

4. APC (aa. 1379-2080) showed a loss of 50\% nuclear fluorescence after 20 seconds of sequential photobleaching of the cytoplasm 